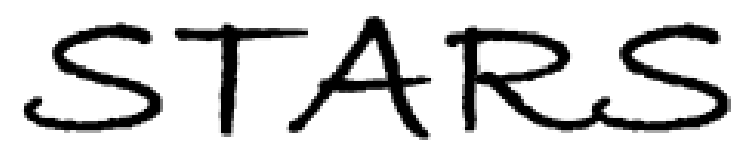

University of Central Florida

STARS

$1-1-2011$

\title{
Discrete beam acceleration in uniform waveguide arrays
}

\author{
Ramy El-Ganainy \\ Konstantinos G. Makris \\ Mohammad Ali Miri \\ University of Central Florida \\ Demetrios N. Christodoulides \\ University of Central Florida \\ Zhigang Chen
}

Find similar works at: https://stars.library.ucf.edu/facultybib2010

University of Central Florida Libraries http://library.ucf.edu

This Article is brought to you for free and open access by the Faculty Bibliography at STARS. It has been accepted for inclusion in Faculty Bibliography 2010 s by an authorized administrator of STARS. For more information, please contactSTARS@ucf.edu.

\section{Recommended Citation}

El-Ganainy, Ramy; Makris, Konstantinos G.; Miri, Mohammad Ali; Christodoulides, Demetrios N.; and Chen, Zhigang, "Discrete beam acceleration in uniform waveguide arrays" (2011). Faculty Bibliography 2010 s. 1281.

https://stars.library.ucf.edu/facultybib2010/1281

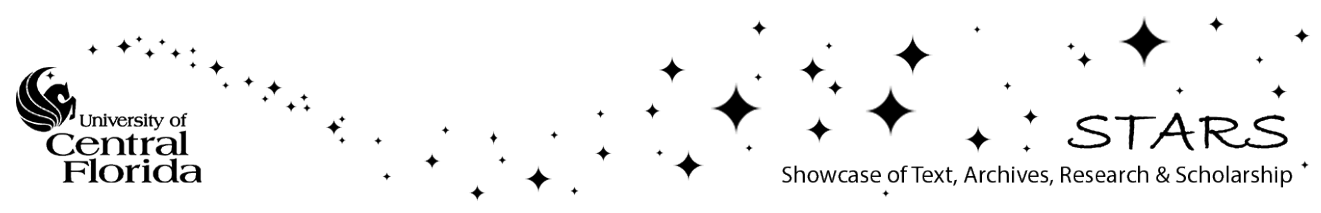




\title{
Discrete beam acceleration in uniform waveguide arrays
}

\author{
Ramy El-Ganainy, ${ }^{1}$ Konstantinos G. Makris, ${ }^{2}$ Mohammad Ali Miri, ${ }^{3}$ Demetrios N. Christodoulides, ${ }^{3}$ and Zhigang Chen ${ }^{4}$ \\ ${ }^{1}$ Department of Physics, University of Toronto, 60 St. George Street, Toronto, Ontario, Canada M5S 1A7 \\ ${ }^{2}$ Institute for Theoretical Physics, Vienna University of Technology, A-1040 Vienna, Austria \\ ${ }^{3}$ College of Optics-CREOL, University of Central Florida, Orlando, Florida 32816, USA \\ ${ }^{4}$ Department of Physics and Astronomy, San Francisco State University, San Francisco, California 94132, USA
}

(Received 11 April 2011; published 25 August 2011)

\begin{abstract}
Within the framework of the tight-binding model we demonstrate that Wannier-Stark states can freely accelerate in uniform optical lattices. As opposed to accelerating Airy wave packets in free space, our analysis reveals that in this case the beam main intensity features self-bend along two opposite hyperbolic trajectories. Two-dimensional geometries are also considered and an asymptotic connection between these Wannier-Stark ladders and Airy profiles is presented.
\end{abstract}

DOI: 10.1103/PhysRevA.84.023842

PACS number(s): 42.25.Hz, 42.82.Et, 42.25.Fx

\section{INTRODUCTION}

Optical waveguide lattices have been a subject of intense study in the past few years [1]. These configurations provide an effective approach to control the propagation dynamics of light in a way that would have been otherwise impossible in the bulk. In the linear regime, waveguide arrays have been used to engineer the diffraction properties of optical wave fronts [1]. Perhaps the best known example of such a linear process is that of discrete diffraction which can be directly described within the framework of the so-called tight-binding approximation. One fascinating aspect of optical lattices is their ability to emulate idealized solid-state systems. This feature was extensively exploited in several works in order to optically demonstrate quantum phenomena that are otherwise hindered in solid-state structures due to many-particle interactions. These include, for example, photonic Bloch oscillations [2,3], Rabi oscillations [4,5], and optical dynamic localization [6,7], just to mention a few. Recently, resonant delocalization and beating Bloch oscillations were also predicted in periodically modulated Bloch lattices [8]. At high enough powers, optical discrete solitons in such structures can also form as nonlinear defects [9].

In an independent and more recent line of study, accelerating optical wave fronts have also attracted considerable attention. In particular, Airy self-bending optical beams have been theoretically predicted [10] and experimentally demonstrated in homogeneous media [11]. These wave packets were shown to propagate without exhibiting any appreciable diffraction up to several Rayleigh lengths. Even more importantly, in the absence of any external optical potentials, their main lobe was found to accelerate along a parabolic trajectory [10]. In subsequent studies, the one-dimensional (1D) nature of this family of nonspreading, accelerating Airy beams enabled the first observation of versatile linear optical bullets [12]. In extreme nonlinear optical settings, self-bending plasma channels were also created and studied in air and other material systems such as water [13]. It is important to note that an ideal diffraction-free Airy beam is not square-integrable and thus requires infinite power. In practice, in order to experimentally overcome this hurdle, the transverse Airy field profile needs to be truncated or apodized [10]. If the apodization is relatively slow, it then allows the beam to retain its primary properties over long distances before diffraction effects eventually take over. Despite the surge of activities in this field, thus far accelerating beams have only been investigated in homogeneous media. It will certainly be important to consider the possibility of such freely accelerating states in periodic environments, such as, for example, waveguide arrays or coupled resonator optical waveguides (CROWs) [14]. While two-dimensional nondiffracting localized beams have previously been analyzed in uniform optical lattices [15], the existence of their 1D counterparts is still an open question, and has not been addressed so far. In this study we demonstrate that Wannier-Stark states can freely accelerate in uniform waveguide lattices. Both $1 \mathrm{D}$ and two-dimensional (2D) configurations are considered in our work. We present analytical results for the acceleration of such discrete beams and we establish their asymptotic connection with optical Airy field profiles. This paper is organized as follows: Sec. II is devoted to investigating the evolution of Wannier-Stark ladders in a uniform waveguide array while the problem is solved from two different approaches. Afterwards an approximate formula is derived describing the curvature of this self-bending beam. Then as a generalization the $2 \mathrm{D}$ case is also investigated. Results are illustrated through relevant examples. In Sec. III, we study the asymptotic behavior of these discrete self-bending beams, and compare it with Airy beams in uniform media.

\section{SELF-BENDING WANNIER STARK LADDERS}

We begin our analysis by examining the acceleration properties of Wannier-Stark ladder states. In doing so we employ a tight-binding model (discrete model) for describing beam interactions in optical waveguide arrays. This description is particularly successful in weakly coupled systems dominated by bound states (first band). In order to identify accelerating beams in such periodic structures, we follow the same arguments previously used in free-space propagation. In this latter case, it is well known that the Airy profile represents a stationary eigenfunction of the Schrödinger equation in the presence of a linear potential. When this potential is turned off, this same Airy eigenfunction remains invariant during propagation while its main lobe follows a parabolic trajectory (self-bends) [16]. On the other hand, in optical waveguide arrays, when a linear optical potential is superimposed on their 
transverse periodic refractive index modulation, the resulting stationary eigenfunctions take the form of a Wannier-Strak ladder [2]. In view of this discussion, the question naturally arises as to the behavior of these profiles when released in a uniform array. In order to answer this question, we consider the normalized discrete diffraction equation with the action of a linear potential:

$$
i \frac{\partial \phi_{n}}{\partial z}+\left(\phi_{n-1}+\phi_{n+1}\right)+n \bar{\alpha} \phi_{n}=0 .
$$

In Eq. (1), $\varphi_{n}$ represents the modal optical field amplitude at site $n, z$ is a normalized propagation coordinate scaled with respect to the coupling constant [2], and $\bar{\alpha}$ is the linear strength in the refractive index modulation. By taking the discrete Fourier transform of Eq. (1) we obtain

$$
i \frac{\partial a(\kappa, z)}{\partial z}+2 \cos (\kappa) a(\kappa, z)+i \bar{\alpha} \frac{\partial a(\kappa, z)}{\partial \kappa}=0 .
$$

Here the Fourier transform pairs are defined as $a(\kappa, z)=\frac{1}{\sqrt{2 \pi}} \sum_{n} \phi_{n}(z) \exp (-i \kappa n) \quad$ and $\quad \phi_{n}(z)=\frac{1}{\sqrt{2 \pi}} \int_{-\pi}^{\pi}$ $a(\kappa, z) \exp (i \kappa n) d \kappa$, respectively. Assuming a stationary solution of the form $\varphi_{n}^{m}=u_{n}^{m} \exp \left(i \beta_{m} z\right)$, the Fourier transform of $u_{n}^{m}$ can be obtained from Eq. (2) and takes the form [2] $\tilde{u}^{m}(\kappa)=\exp \left\{-\frac{i}{\bar{\alpha}}[m \bar{\alpha} \kappa-2 \sin (\kappa)]\right\}$ where $m$ is an integer. Note that changing $m$ only shifts the location of the Wannier-Stark (WS) eigenmode without changing its profile. Hence, without any loss of generality we proceed by setting $m$ to zero. In the real domain, the WS mode takes the form [2] $u_{n}^{0}=\sqrt{2 \pi} J_{-n}(2 / \bar{\alpha})$. We now consider a uniform array with $\bar{\alpha}=0$ and we use WS profile as an input, and we investigate the resulting propagation dynamics under these conditions. For the uniform array, Eq. (1) reads $i \frac{\partial \phi_{n}}{\partial z}+\left(\phi_{n-1}+\phi_{n+1}\right)=0$ and Eq. (2) becomes $i \frac{\partial a(\kappa, z)}{\partial z}+2 \cos (\kappa) a(\kappa, z)=0$. From this last equation, any initial condition $a(\kappa, 0)$ will evolve according to

$$
a(\kappa, z)=a(\kappa, 0) \exp [2 i z \cos (\kappa)] .
$$

Starting with the WS profile $a(\kappa, 0)=\exp \left[\frac{2 i}{\alpha} \sin (\kappa)\right]$ (corresponding to $\left.u_{n}^{0}\right)$, we find that $a(\kappa, z)=$ $\exp \left\{2 i\left[\frac{1}{\alpha} \sin (\kappa)+z \cos (\kappa)\right]\right\}$. The last expression can be written as $a(\kappa, z)=\exp \{2 i \Omega(z) \sin [\kappa+\theta(z)]\}$, where $\Omega(z)=\sqrt{z^{2}+1 / \alpha^{2}}$ and $\theta(z)=\tan ^{-1}(\alpha z)$. By taking the inverse Fourier transform we find that

$$
\phi_{n}(z)=\frac{1}{\sqrt{2 \pi}} \int_{-\pi}^{\pi} \exp \{2 i \Omega(z) \sin [\kappa+\theta(z)]\} \exp (i \kappa n) d \kappa .
$$

A linear change of variable $\kappa=\kappa_{1}-\theta(z)$ yields

$\phi_{n}(z)=\frac{\exp (-i \theta n)}{\sqrt{2 \pi}} \int_{\pi+\theta}^{\pi+\theta} \exp \left[2 i \Omega(z) \sin \left(\kappa_{1}\right)\right] \exp \left(i \kappa_{1} n\right) d \kappa_{1}$.

By using $J_{-n}(2 x)=\frac{1}{2 \pi} \int_{\pi+\theta}^{\pi+\theta} \exp \left[2 i x \sin \left(\kappa_{1}\right)\right] \exp \left(i \kappa_{1} n\right)$ $d \kappa_{1}$ we finally obtain

$$
\phi_{n}(z)=\sqrt{2 \pi} \exp \left[-i n \tan ^{-1}(\alpha z)\right] J_{-n}\left(2 \sqrt{z^{2}+1 / \alpha^{2}}\right) .
$$

Equation (6) shows that upon propagation the WS ladder diffracts in a self-similar way (maintains its mathematical
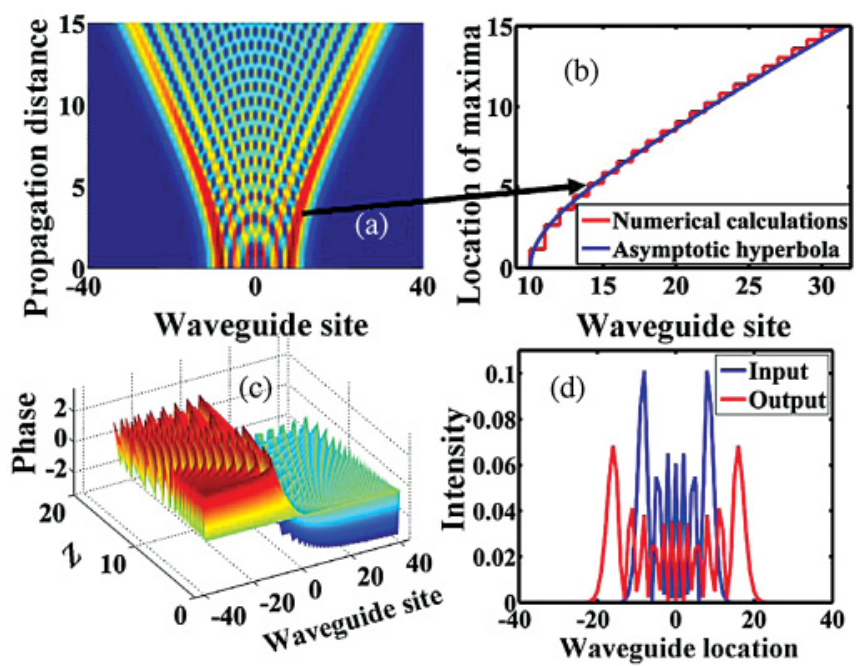

FIG. 1. (Color online) (a) Propagation of WS mode in a uniform lattice with $\alpha=0.2$ and (b) comparison between results obtained from Eq. (6) (red zigzag line) with the asymptotic form of Eq. (7) (blue line). (c) Phase evolution of the transverse field as a function of the propagation distance $z$. (d) Transverse intensity profile at the input [narrow profile with higher peak intensity (blue line)] and at $z=15 / 2$ [diffracted beam with lower peak intensity (red line)]. All physical quantities are normalized.

form). We also observe that the phase of such a mode acquires some tilting that is linear with $n$ during propagation. Figure 1(a) shows the propagation dynamics of a WS ladder centered at the center of an array. For illustration purposes, the parameter $\alpha$ in this simulation was chosen to be $\alpha=$ 0.2. The mode self-similarity is evident from the figure. Moreover, it is clear that the main lobe does not follow a straight line. In order to characterize the curvature of this trajectory we use the asymptotic expansion of the zeros of the Bessel function derivatives. This expansion determines the location of the first maxima and for $J_{n}(x)$ is given by [17] $x_{\max }=n+0.8086165 n^{1 / 3}+0.07249 n^{-1 / 3}+\cdots$. Since we are interested in the maximum values of $\left|J_{n}(x)\right|$, we can use this same expression even for negative values of $n$. From Fig. 1(a) we note that the maximum value of the input happens to be around $n=9$. For such large values of $n$, we can use only the first term in the expansion. By noting that the condition for $\max \left[\left|J_{n}(x)\right|\right]$ is the same as for $\max \left[\left|J_{-n}\left(2 \sqrt{z^{2}+1 / \alpha^{2}}\right)\right|\right]$, the main lobe's trajectory takes the form

$$
n= \pm 2 \sqrt{z_{\max }^{2}+1 / \alpha^{2}}
$$

In formula (7), $z_{\max }$ is the location of the maximum along the propagation direction in waveguide number $n$. From Eq. (7) we observe that the path followed by the main lobe is described by a hyperbola. Figure 1(b) depicts a comparison of the propagation trajectory associated with the maximum of the beam [directly obtained from Eq. (6)] with that predicted by expression (7). Excellent agreement is clearly observed. It is important to mention here that unlike an ideal Airy beam in free space, the WS ladder has finite energy and its intensity is symmetric with respect to its center (even though the field profile is not symmetric). We also note the existence of two main lobes, accelerating in opposite directions. Evidently, 
as the beam further propagates, the trajectory asymptotically becomes linear. Figure 1(c) depicts the antisymmetric phase behavior calculated from Eq. (6), while Fig. 1(d) shows the input-output beam transverse profiles. We stress the fact that, like its Airy wave counterpart, the center of mass of this beam remains invariant upon propagation $[10,11,18]$. Note that the hyperbolic trajectory is not a result of any asymmetric refractive index distribution, but rather a direct outcome of interference effects. This can be better understood by rederiving Eq. (6) through the impulse response of a uniform array. In other words, we can arrive at this same result using the convolution summation $\phi_{n}(z)=\sum_{k=-\infty}^{\infty} i^{(n-k)} u_{k}^{0} J_{n-k}(2 z)$, where $i^{n} J_{n}(2 z)$ is the impulse response for a $\delta$ excitation in the middle channel $u_{k}^{0}=\delta_{k, 0}$. If we now insert a WS mode as an input, we obtain $\phi_{n}(z)=\sqrt{2 \pi} \sum_{k=-\infty}^{\infty} i^{(n-k)} J_{-k}(2 / \alpha) J_{n-k}(2 z)$. Substituting $n-k=q$, we find

$$
\phi_{n}(z)=\sqrt{2 \pi} \sum_{q=-\infty}^{\infty} \exp (i q \pi / 2) J_{q-n}(2 / \alpha) J_{q}(2 z),
$$

where $i=\exp (i \pi / 2)$ was used. In order to proceed, we exploit Gegenbauer's theorem for Bessel functions of the first kind [19]:

$$
\begin{aligned}
& J_{m}(w) \cos (m \chi)=\sum_{k=-\infty}^{\infty} J_{m+k}(u) J_{k}(v) \cos (k \vartheta), \\
& J_{m}(w) \sin (m \chi)=\sum_{k=-\infty}^{\infty} J_{m+k}(u) J_{k}(v) \sin (k \vartheta) .
\end{aligned}
$$

Multiplying Eq. (9) with the complex number $i=\sqrt{-1}$ and adding the result to Eq. (9), we obtain

$$
J_{m}(w) \exp (i m \chi)=\sum_{k=-\infty}^{\infty} J_{m+k}(u) J_{k}(v) \exp (i k \vartheta) .
$$

In Eqs. (9) and (10), $w=\sqrt{u^{2}+v^{2}-2 u v \cos (\vartheta)}, u-$ $v \cos (\vartheta)=w \cos (\chi)$, and $v \sin (\vartheta)=w \sin (\chi)$. The right-hand side of Eqs. (8) and (10) are identical (apart from a factor of $\sqrt{2 \pi}$ ) provided that $\vartheta=\pi / 2, u=2 / \alpha$, and $v=2 z$. Under these conditions $w=\sqrt{u^{2}+v^{2}}=2 \sqrt{z^{2}+1 / \alpha^{2}}$ and $\chi=$ $\tan ^{-1}(v / u)=\tan ^{-1}(\alpha z)$, and hence Eq. (8) formally reduces to that of Eq. (6). Interpreting the self-bending as an interference phenomenon provides a means to synthesize special beams with desired properties. For example, one might be interested in concentrating all the beam's energy in only one lobe instead of two, in which case we can superimpose two shifted WS modes of the form $\varphi_{n}(0)=\sqrt{2 \pi}\left[J_{n}(2 / \alpha)+J_{n-1}(2 / \alpha)\right]$. Figure 2(a) shows the intensity profile of such input where it is clear that the left intensity peak was suppressed. Figure 2(b) depicts the evolution of this asymmetric profile beam as it propagates through the array. The path followed by the right main lobe is not much affected; in other words, it is still a hyperbola. We also note that distinct diffraction patterns emerge as different WS ladder superpositions are used. In Fig. 2(b) traces of the suppressed left peak intensity are observed after some propagation distance. This is a direct outcome of the linear nature of the problem and the antisymmetric behavior of the phase. It is important to mention
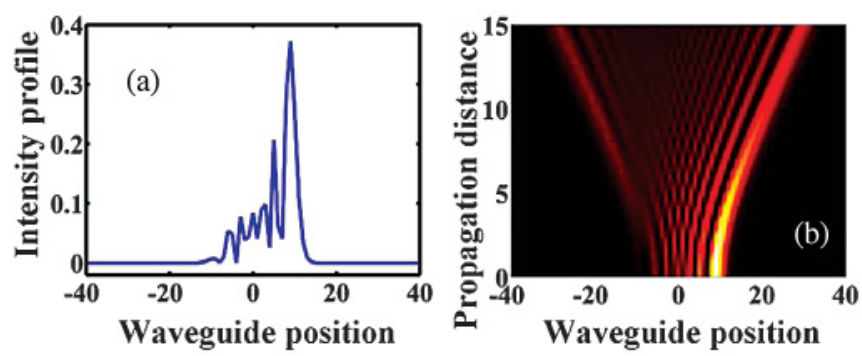

FIG. 2. (Color online) (a) Dimensionless intensity profile of two superimposed WS ladders shifted by one site from each other and (b) the propagation dynamics of such an input optical beam. Note the existence of a prominent lobe at the input.

that the predicted self-bending can be experimentally observed using experimentally realizable optical lattices. For example, in an optical waveguide array made of 40 elements and having a coupling constant equal to $50 \mathrm{~cm}^{-1}$, a propagation distance of $1 \mathrm{~mm}$ will be needed for the main lobes of a WS mode with $\alpha=0.25$ to experience a shift of 5 waveguide sites.

These results can be directly extended to the twodimensional case where the discrete evolution equation can be written as

$$
i \frac{\partial \phi_{n, l}}{\partial z}+\left(\phi_{n-1, l}+\phi_{n+1, l}+\phi_{n, l-1}+\phi_{n, l+1}\right)=0 .
$$

If the input profile can be decomposed into the product $\varphi_{n, l}(0)=u_{n}(0) w_{l}(0)$, then Eq. (11) is separable, i.e., $\varphi_{n, l}(z)=$ $u_{n}(z) w_{l}(z)$. In other words, starting with a 2D WS ladder of the form $\varphi_{n, l}=2 \pi J_{-n}\left(2 / \alpha_{x}\right) J_{-l}\left(2 / \alpha_{y}\right)$, then after a propagation distance $z$, the output intensity is given by

$$
\left|\phi_{n, l}(z)\right|^{2}=4 \pi^{2} J_{-n}^{2}\left(2 \sqrt{z^{2}+1 / \alpha_{x}^{2}}\right) J_{-l}^{2}\left(2 \sqrt{z^{2}+1 / \alpha_{y}^{2}}\right) .
$$

Figure 3 shows the input and output intensity profiles for $\alpha_{x}=0.2$ and $\alpha_{y}=0.1$ after a normalized propagation distance of $z=50$. Note that due to the anisotropy of the parameter $\alpha_{x, y}$ the self-bending rate in each direction is different, which is clearly manifested in the change of the aspect ratio of the input profile during propagation (see Fig. 3).
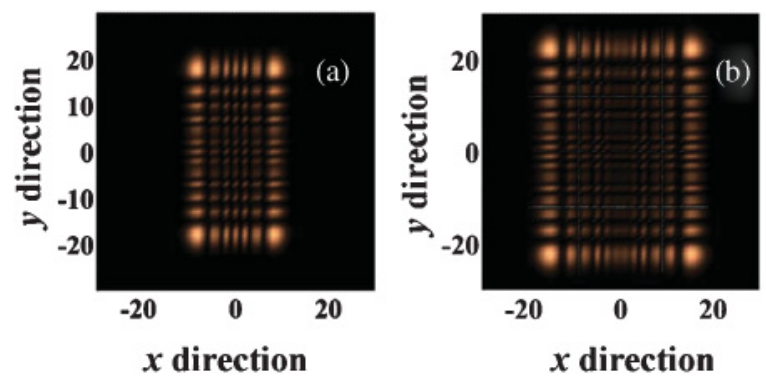

FIG. 3. (Color online) Two-dimensional normalized (a) input and (b) output beam intensities for the parameters mentioned in the text. Darker regions represent lower intensities. 


\section{ASYMPTOTIC RELATION BETWEEN WS LADDER AND THE AIRY PROFILE}

In this section we investigate how the WS and the Airy modes relate, if at all. We begin by highlighting the differences between these two wavefronts. As it has been mentioned previously, the Airy profile is a stationary solution of the continuous paraxial wave equation in the presence of a linear potential, while the WS is an eigenfunction of a lattice when its effective refractive index varies linearly with the site index $n$. In the former case, the eigenfunction is asymmetric and contains infinite energy while the latter regime the intensity profile is symmetric and its power content is finite. In terms of propagation dynamics, ideal Airy beams follow a parabolic trajectory and are diffraction-free [10]. On the other hand, the WS profiles experience diffraction and their main lobes move on hyperbolic curves. In the light of these differences, at first sight it might appear that there should be no connection between these two families of eigenmodes. However, a closer examination of the paraxial equation of diffraction leads to a different conclusion. To establish this similarity let us consider the normalized paraxial equation of diffraction in the presence of a linear refractive index potential, e.g.,

$$
i \frac{\partial \psi}{\partial z}+\frac{\partial^{2} \psi}{\partial x^{2}}+\gamma x \psi=0
$$

By using a finite difference grid to approximate the second partial derivative $\partial_{x x} \psi=\left(\psi_{n+1}+\psi_{n-1}-2 \psi_{n}\right)$ $/(\Delta x)^{2}$, we obtain $i \frac{\partial \psi_{n}}{\partial z}+\frac{\psi_{n+1}+\psi_{n-1}-2 \psi_{n}}{(\Delta x)^{2}}+n \gamma \Delta x \psi_{n}=0$. Furthermore, by using the scale $\eta=z /(\Delta x)^{2}$, the previous equation reduces to $i \partial_{\eta} \psi_{n}+\psi_{n+1}+\psi_{n-1}-$ $2 \psi_{n}+n \gamma(\Delta x)^{3} \psi_{n}=0 . \quad$ A gauge transformation of the form $\psi_{n}=\varphi_{n} \exp (-2 i \eta)$ finally yields

$$
i \frac{\partial \phi_{n}}{\partial \eta}+\left(\phi_{n-1}+\phi_{n+1}\right)+n \alpha \phi_{n}=0,
$$

where $\gamma(\Delta x)^{3}=\alpha$. Equation (14) is exactly the same as Eq. (1) previously used to derive the WS modes, and at the same time it represents an approximation to the diffraction equation that has an Airy function solution in the limit $\Delta x \rightarrow 0$ or $\alpha \rightarrow 0$. This simple analysis strongly hints toward a relationship between both the continuous and the discrete models. Yet at this point, it is not clear how such a symmetric WS profile (in intensity) should asymptotically approach an asymmetric intensity distribution (Airy). In order to resolve this apparent contradiction, we study the behavior of the WS modes described by Eq. (6) as the parameter $\alpha$ approaches zero. As was shown in Fig. 1(d) increasing the argument of the WS function [corresponding to decreasing $\alpha$ in Eq. (6)] will shift the two main intensity peaks further apart to the left and right. From Eq. (7), the positions of the peaks can be approximated by $n \approx \pm 2 / \alpha$ and evidently the locations of the lobes approach $-\infty$ and $\infty$ as $\alpha \rightarrow 0$. In order to proceed with the analysis, we use the integral definition of a Bessel function, $J_{-n}(2 / \alpha)=\frac{1}{\pi} \int_{0}^{\pi} \cos [n t+(2 / \alpha) \sin (t)] d t$. Figures 4(a) and 4(b) show a plot of the cosinusoidal function in the two different limits $\alpha \rightarrow 0$ and $n \approx \mp 2 / \alpha \rightarrow \mp \infty$, respectively. Note that the two curves are plotted for different ranges of $t$. From Fig. 4(a), when $n \rightarrow-\infty$, one can see it is
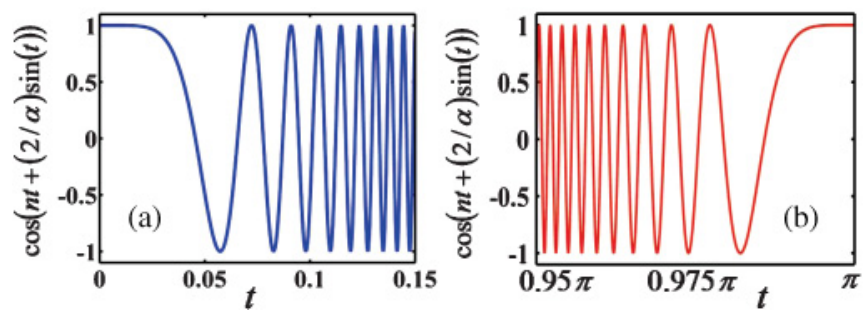

FIG. 4. (Color online) Plot of the function $\cos [n t+(2 / \alpha) \sin (t)]$ for $\alpha=2 \times 10^{-5}$ for the two different limits (a) $n=-2 / \alpha$ and (b) $n=2 / \alpha$.

sufficient to carry out the integral in the vicinity $t \approx 0$ while for the case of $n \rightarrow \infty$, the dominant contribution is mainly due to the integrand at $t \approx \pi$. This type of argument is justified since the integral kernel oscillates very quickly outside the mentioned ranges and hence their contributions cancel out. Let us first focus on the left-hand peak where $n \rightarrow-\infty$. Under these conditions, the WS mode $J_{-n}(2 / \alpha)$ can be written as

$$
J_{-n}(2 / \alpha) \cong \frac{1}{\pi} \int_{0}^{\pi} \cos \left[(n+2 / \alpha) t-t^{3} /(3 \alpha)\right] d t .
$$

where the Taylor series expansion $\sin (t) \approx t-t^{3} / 3$ ! was used. Note that, similar to stationary phase approximations, the limit of the integration in Eq. (15) were kept the same. A change of variable $t / \sqrt[3]{\alpha}=s$ gives

$J_{-n}(2 / \alpha) \cong \frac{\sqrt[3]{\alpha}}{\pi} \int_{0}^{\pi / \sqrt[3]{\alpha}} \cos \left[\sqrt[3]{\alpha}(n+2 / \alpha) s-s^{3} / 3\right] d s$.

Since $\alpha \rightarrow 0$, it follows that $\pi / \sqrt[3]{\alpha} \rightarrow \infty$ and the integral in Eq. (16) becomes that associated with an Airy function. Thus in this limit, the WS mode can be written as

$$
J_{-n}(2 / \alpha) \cong \frac{\sqrt[3]{\alpha}}{\pi} \operatorname{Ai}[-\sqrt[3]{\alpha}(n+2 / \alpha)],
$$

which is valid only when $\alpha \rightarrow 0$ and $n \approx-2 / \alpha \rightarrow-\infty$. The asymptotic formula (17) reveals that as we approach the continuum limit, the WS mode at $x \rightarrow-\infty$ will locally approach the Airy profile. Figure 5 depicts a plot of the WS mode calculated from the Airy expression of Eq. (17) for $\alpha=0.0002$ versus the exact values obtained directly using Bessel functions where an excellent matching is observed. Our analysis so far predicts that one branch (left side) of the WS mode will indeed approach an Airy beam profile; however, it

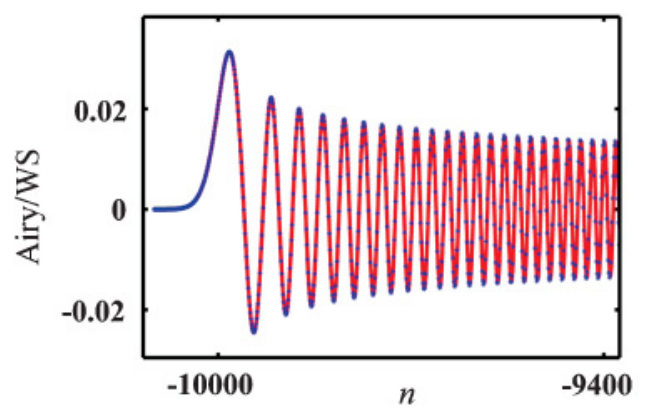

FIG. 5. (Color online) Exact Wannier Stark profile with $\alpha=$ 0.0002 (dotted blue line) versus the asymptotic expression obtained from Eq. (17) shown in red continuous line. 
does not offer an answer yet as to how an asymmetric intensity distribution becomes the asymptotic limit of a symmetric mode. To resolve this issue we must investigate the behavior of the opposite branch (right side) of the WS mode. When $n \approx 2 / \alpha \rightarrow \infty$ we have

$$
J_{-n}(2 / \alpha)=\frac{1}{\pi} \int_{0}^{\pi} \cos [n(\pi-\tau)+(2 / \alpha) \sin (\pi-\tau)] d \tau,
$$

where the coordinate transformation $\pi-t=\tau$ was used. Equation (18) can be further reduced to $J_{-n}(2 / \alpha)=$ $\frac{1}{\pi} \int_{0}^{\pi} \cos [n \pi-n \tau+(2 / \alpha) \sin (\tau)] d \tau$. By using trigonometric identities and a Taylor expansion around $\tau=0$ (equivalent to expanding around $t=\pi)$ we obtain $J_{-n}(2 / \alpha)=$ $\frac{(-1)^{n}}{\pi} \int_{0}^{\pi} \cos \left[(n-2 / \alpha) \tau+\tau^{3} /(3 \alpha)\right] d \tau$. As before, we use the substitution $\tau / \sqrt[3]{\alpha}=s$ to get

$$
\begin{aligned}
J_{-n}(2 / \alpha)= & \frac{(-1)^{n} \sqrt[3]{\alpha}}{\pi} \\
& \times \int_{0}^{\pi / \sqrt[3]{\alpha}} \cos \left(\sqrt[3]{\alpha}(n-2 / \alpha) s+s^{3} / 3\right) d s .
\end{aligned}
$$

Again the integral in Eq. (19) represents an Airy function and in the limit $\alpha \rightarrow 0$ and $\pi / \sqrt[3]{\alpha} \rightarrow \infty$, i.e.,

$$
J_{-n}(2 / \alpha)=\frac{(-1)^{n} \sqrt[3]{\alpha}}{\pi} \operatorname{Ai}[\sqrt[3]{\alpha}(n-2 / \alpha)]
$$

Equation (20) indicates that in this case the field amplitude changes sign along neighboring points on the discretization grid. In other words, even though the intensity is that of an Airy beam, the field itself is staggered ( $\pi$ out of phase between adjacent channels), having no analog in the continuous limit. Thus Eq. (20) is a solution only in the discrete limit and by no means satisfies the differential equation of diffraction. In other words, in the continuous limit, only the left side of the WS mode physically corresponds to an Airy wave packet while the right branch is staggered because of its momentum proximity to the photonic band gap.

At this point, it is worth comparing the acceleration of the asymptotic Airy profile of Eq. (17) with that predicted by formula (7). Consider the function $f=\operatorname{Ai}[-\sqrt[3]{\alpha}(n+2 / \alpha)]$; using the relation $\gamma(\Delta x)^{3}=\alpha$ gives
$f=\operatorname{Ai}(-\sqrt[3]{\gamma} \Delta x n-2 \sqrt[3]{\alpha} / \alpha)$. By substituting $\Delta x n=x$, we obtain $f(x)=\operatorname{Ai}(-\sqrt[3]{\gamma} x-2 \sqrt[3]{\alpha} / \alpha)$. The first term in the argument of $f$ gives rise to a transverse Airy profile while the second term is just a shift. Such an input profile, upon propagation in free space, will evolve into [10] $f(x, z)=$ $\operatorname{Ai}\left(-\sqrt[3]{\gamma} x-2 \sqrt[3]{\alpha} / \alpha-\sqrt[3]{\gamma^{4}} z^{2}\right)$, where $z$ is the propagation distance. A backward transformation to discrete coordinates, i.e., using $\gamma(\Delta x)^{3}=\alpha$ and $\eta=z /(\Delta x)^{2}$, results in

$$
f(n, \eta)=\operatorname{Ai}\left(-\sqrt[3]{\gamma} \Delta x n-2 \sqrt[3]{\alpha} / \alpha-\sqrt[3]{\gamma^{4}}(\Delta x)^{4} \eta^{2}\right) .
$$

The acceleration is obtained by setting the argument of Eq. (21) equal to zero, yielding $\sqrt[3]{\alpha}\left(n+2 / \alpha+\alpha \eta^{2}\right)=0$, or in other words, we find that $n=-\left(2 / \alpha+\alpha \eta^{2}\right)$. Similarly the other branch will evolve according to $n=2 / \alpha+\alpha \eta^{2}$. In summary, in this limit, the main two lobes follow a parabolic trajectory:

$$
n= \pm\left(2 / \alpha+\alpha \eta^{2}\right)
$$

These same results can be readily obtained from Eq. (7) by using a Taylor series expansion in the limit of very small $\alpha$ [with $z_{\max }$ playing the same role as $\eta$ in Eq. (22)]. These results establish the inherent similarity between Airy beams and Wannier-Stark states in optical lattices.

\section{CONCLUSIONS}

In conclusion, we have presented analytical results concerning the propagation dynamics of Wannier-Stark ladders in uniform waveguide arrays and showed that the two main lobes of such a mode experience self-bending along opposite trajectories. Unlike free space Airy beams, the WS modes are actually diffracting and their self-bending trajectory is hyperbolic as opposed to being parabolic. Two-dimensional analytical solutions of WS modes were also found. In the asymptotic limit we also studied the inherent relationship between the WS ladders and the Airy profiles. By exploiting the connection between continuous variables and discrete representations, we were able to show that one branch of the WS solutions asymptotically approaches the Airy function while in the same limit the other side becomes nondifferentiable and hence does not qualify as a mathematically valid solution to the paraxial equation of diffraction.
[1] D. N. Christodoulides, F. Lederer, and Y. Silberberg, Nature 424, 817 (2003).

[2] U. Peschel, T. Pertsch, and F. Lederer, Opt. Lett. 23, 1701 (1998).

[3] T. Pertsch, P. Dannberg, W. Elflein, A. Bräuer, and F. Lederer, Phys. Rev. Lett. 83, 4752 (1999).

[4] K. G. Makris, D. N. Christodoulides, O. Peleg, M. Segev, and D. Kip, Opt. Express 16, 10309 (2008).

[5] K. Shandarova, C. E. Rüter, D. Kip, K. G. Makris, D. N. Christodoulides, O. Peleg, and M. Segev, Phys. Rev. Lett. 102, 123905 (2009).

[6] G. Lenz, I. Talanina, and C. M. de Sterke, Phys. Rev. Lett. 83, 963 (1999).
[7] S. Longhi, M. Marangoni, M. Lobino, R. Ramponi, P. Laporta, E. Cianci, and V. Foglietti, Phys. Rev. Lett. 96, 243901 (2006).

[8] R. El-Ganainy, D. N. Christodoulides, C. R. Rüter, and D. Kip, Opt. Lett. 36, 1464 (2011).

[9] D. N. Christodoulides and R. I. Joseph, Opt. Lett. 13, 794 (1988)

[10] G. A. Siviloglou and D. N. Christodoulides, Opt. Lett. 32, 979 (2007).

[11] G. A. Siviloglou, J. Broky, A. Dogariu, and D. N. Christodoulides, Phys. Rev. Lett. 99, 213901 (2007).

[12] A. Chong, W. H. Renninger, D. N. Christodoulides, and F. W. Wise, Nat. Photonics 4, 103 (2010). 
[13] P. Polynkin, M. Kolesik, J. V. Moloney, G. A. Siviloglou, and D. N. Christodoulides, Science 324, 229 (2009).

[14] A. Yariv, Y. Xu, R. K. Lee, and A. Scherer, Opt. Lett. 24, 711 (1999).

[15] O. Manela, M. Segev, and D. N. Christodoulides, Opt. Lett. 30, 2611 (2005).

[16] M. V. Berry and N. L. Balazs, Am. J. Phys. 47, 264 (1979).
[17] Handbook of Mathematical Functions, edited by M. Abramowitz and I. A. Stegun, (Dover, New York, 1965), p. 371, formula 9.5.16.

[18] I. M. Besieris, and A. M. Shaarawi, Opt. Lett. 32, 2447 (2007).

[19] Handbook of Mathematical Functions, edited by M. Abramowitz and I. A. Stegun (Dover, New York, 1965), p. 363, formula 9.1.79. 